\title{
An emergency response to the opioid overdose crisis in Canada: a regulated opioid distribution program
}

\author{
Mark Tyndall MD ScD
}

Cite as: CMAJ 2018 January 15;190:E35-6. doi: 10.1503/cmaj.171060

$\mathbf{U}$ nintentional drug overdoses have rapidly become a public health crisis in Canada. Collecting accurate numbers remains problematic, but it is estimated that 2458 overdose deaths occurred in 2016. ${ }^{1}$ In the province of British Columbia - ground zero for the epidemic - more than 1000 overdose deaths were reported in the first eight months of 2017. ${ }^{2}$ Although the rates of overdoses vary across the country, no regions have been spared, and communities should be bracing for the worst.

The overdose crisis has exposed the large gaps in our drug treatment systems and the uneven access to harm reduction services. Even in $\mathrm{BC}$, the monthly death toll has remained stubbornly unchanged, despite the declaration of a public health emergency in April 2016, a rapid scale-up of community-based naloxone distribution, intensified public education campaigns, expansion of opioid agonist therapy and the establishment of overdose prevention units.

At the core of the current crisis is a drug supply that has become contaminated with potent synthetic opioids, mainly in the form of fentanyl and carfentanil. Although communities have periodically experienced increased numbers of overdose deaths owing to transient changes in drug supply, it appears that these highly toxic drugs are here for the foreseeable future. Testing from border seizures and confiscated street drugs, postmortem toxicology and front-line drug testing has confirmed that fentanyl and other powerful opioid analogs are pervasive. ${ }^{3}$ These cheap and easily produced synthetic compounds have replaced illegal heroin. Simply warning people to avoid fentanyl or the plethora of new synthetic analogs is both naive and ineffective.

Also contributing directly to the death toll is a renewed focus on reducing opioid prescribing by physicians. ${ }^{4}$ Although it is widely accepted that liberal prescribing practices have contributed to the current crisis, what to do about it is not clear. Encouraging physicians to reduce their opioid prescriptions in an environment where the illegal alternatives are lethal is harmful. Abruptly cutting people off their prescription will likely lead to withdrawal. A reduction in the overall number of prescriptions creates a shrinking supply of diverted drugs, the unintended consequence of which may be to

\section{KEY POINTS}

- The rapid rise of unintentional drug overdoses in Canada is directly related to the introduction of potent synthetic opioids into the illegal drug market.

- People who once had a secure source of pharmaceuticalgrade opioids or illegal heroin are now using drugs that are extremely potent and have unpredictable effects.

- Naloxone distribution, harm reduction programs and expanded access to drug treatment are critical first responses, but have limitations in reach and impact.

- A regulated, low-barrier distribution of pharmaceutical-grade opioids is a scalable intervention that could reduce harms and save lives.

push people, many of whom were not even known to be chronic opioid users, into much more unstable and dangerous drug markets. ${ }^{5}$ People who once had consistent access to either prescribed or diverted pharmaceutical opioids are suddenly in grave danger of being poisoned by a single lethal purchase.

We are now faced with the very real possibility of a protracted epidemic of overdose deaths that encompasses an ever-widening swath of the Canadian population, many of whom fall outside our usual targets for harm reduction programs. Although a number of important "upstream" interventions are critical to our response, including a functioning system for treating addiction, supportive housing programs, screening programs for at-risk youth, a cultural shift in how we view drug users and a reduced reliance on the criminal justice system, ${ }^{1}$ these will come too late for the families who will lose a loved one this year or the next. We cannot simply give up on the current group of chronic opioid users who are playing a form of "Russian roulette" with every injection or inhalation.

The public health response to any poisoning epidemic should be to provide safer alternatives for people at risk. In the case of the overdose crisis, this would mean providing a regulated supply of pharmaceutical-grade opioids to people at highest risk of overdose. Any options to expand access to pharmaceutical-grade 
opioids runs counter to prevailing narratives regarding the origins of the current overdose crisis. However, it is now clear that the rapid rise of overdose deaths across the country is a result of illegal, unregulated and lethal synthetic compounds that have largely replaced the regular street supply. ${ }^{3}$

A number of models could be rolled out. A well-established program is the Crosstown Clinic in Vancouver, where about 140 people receive injectable heroin or hydromorphone under medical supervision. ${ }^{6}$ The effectiveness of this program is supported by the rigorous scientific evaluation coming out of the Study to Assess Longerterm Opioid Medication Effectiveness (SALOME) trial, as well as several other heroin-assisted therapy trials. ${ }^{7}$ However, the program has limitations with respect to scale-up, owing to the high cost of injectable hydromorphone, the infrastructure requirements and that participants must attend a medical clinic up to three times per day. In an effort to expand access to injectable opioid agonist therapy, a guideline has been developed to support new models for distribution that aim to lower both the barriers to qualify and the requirements to participate. ${ }^{8}$

Another important initiative - the dispensing of slow-release oral morphine - comes from a new guideline that was recently released in $\mathrm{BC} .{ }^{9}$ This medication can be taken once a day in a dose corresponding to the needs of the individual; oral ingestion is encouraged over injection. ${ }^{10}$ This could be an attractive option for some, but others are not interested in a sustained-release formulation, and still others want to inject their drugs.

A compelling argument could be made for an untested model that sees hydromorphone pills, which are cheap and readily available, being made widely available to dependent individuals. Most people who received these pills would ultimately crush, filter and inject the hydromorphone. The distribution points could include community clinics, supportive housing units, pharmacies, special harm-reduction units and supervised injection sites. Scale-up would be straightforward and this model would appeal especially to the large number of opioid users who already use pill formulations. It would probably also attract people who are not likely to access harm reduction programs. As this would be the lowest-barrier program, there would be an increased likelihood for diversion, and protocols would be necessary to ensure that the program participants were the people who received the drugs. As with all drug distribution programs, participants would have access to a range of wraparound services that would allow them to transition to other treatments.

Developing a regulated opioid distribution program would require some regulatory changes and a comprehensive monitor- ing and evaluation strategy, along with a general shift in how we approach the epidemic of opioid overdose. However, we will not get out of this crisis by simply improving on current prevention strategies. We must address head-on the direct cause of the overdose epidemic: a toxic drug market.

\section{References}

1. National report: apparent opioid-related deaths (2016). Ottawa: Government of Canada; 2017. Available: www.canada.ca/en/health-canada/services /substance-abuse/prescription-drug-abuse/opioids/national-report-apparent -opioid-related-deaths.html (accessed 2017 Dec. 5).

2. Illicit drug overdose deaths in BC: January 1, 2007-September 30, 2017. Burnaby (BC): Office of the Chief Coroner, Ministry of Public Safety \& Solicitor General; 2017. Available: www2.gov.bc.ca/assets/gov/public-safety-and-emergency-services/ death-investigation/statistical/illicit-drug.pdf (accessed 2017 Nov. 9).

3. Fentanyl-detected illicit drug overdose deaths: January 1, 2012 to September 30, 2017. Burnaby (BC): Office of the Chief Coroner, Ministry of Public Safety \& Solicitor General; 2017. Available: www2.gov.bc.ca/assets/gov/public-safety-and -emergency-services/death-investigation/statistical/fentanyl-detected-overdose. pdf (accessed 2017 Nov. 9).

4. Busse JW, Craigie S, Juurlink DN, et al. Guideline for opioid therapy and chronic noncancer pain. CMAJ 2017;189:E659-66.

5. Faryar KA, Freeman CL, Persaud AK, et al. The effects of Kentucky's comprehensive opioid legislation on patients presenting with prescription opioid or heroin abuse to one urban emergency department. J Emerg Med 2017;53: 805-14.

6. Oviedo-Joekes E, Guh D, Brissette S, et al. Hydromorphone compared with diacetylmorphine for long-term opioid dependence: a randomized clinical trial. JAMA Psychiatry 2016;73:447-55.

7. Palis H, Marchand K, Guh D, et al. Men's and women's response to treatment and perceptions of outcomes in a randomized controlled trial of injectable opioid assisted treatment for severe opioid use disorder. Subst Abuse Treat Prev Policy 2017;12:25.

8. Supervised Injectable Opioid Agonist Treatment Guidance Committee. Guidance for injectable opioid agonist treatment for opioid use disorder. Vancouver: Ministry of Health, British Columbia, and British Columbia Centre on Substance Use; 2017. Available: www.bccsu.ca/wp-content/uploads/2017/10/ BC-iOAT-Guidelines-10.2017.pdf (accessed 2017 Nov. 20).

9. Provincial Opioid Use Disorder Treatment Guideline Committee. A guideline for the clinical management of opioid use disorder. Vancouver: Ministry of Health, British Columbia, and British Columbia Centre on Substance Use; 2017. Available: www.bccsu.ca/wp-content/uploads/2017/06/BC-OUD-Guidelines_ June2017.pdf (accessed 2017 Oct. 20).

10. Ferri M, Minozzi S, Bo A, et al. Slow-release oral morphine as maintenance therapy for opioid dependence. Cochrane Database Syst Rev 2013;(6):CD009879.

\section{Competing interests: None declared.}

This article has been peer reviewed.

Affiliations: BC Centre for Disease Control, Vancouver, BC; School of Population and Public Health, University of British Columbia, Vancouver, $\mathrm{BC}$

Correspondence to: Mark Tyndall, mark.tyndall@bccdc.ca 